\title{
First case of bilateral, synchronous anaplastic variant of spermatocytic seminoma treated with radical orchifunicolectomy as single approach: Case report and review of the literature
}

\author{
Giorgio Gentile ${ }^{1}$, Francesca Giunchi ${ }^{2}$, Riccardo Schiavina ${ }^{1}$, Alessandro Franceschelli ${ }^{3}$, \\ Marco Borghesi ${ }^{1}$, Ziv Zukerman ${ }^{1}$, Matteo Cevenini ${ }^{1}$, Valerio Vagnoni ${ }^{1}$, Daniele Romagnoli ${ }^{1}$, \\ Fulvio Colombo ${ }^{3}$, Giuseppe Martorana ${ }^{1}$, Eugenio Brunocilla ${ }^{1}$ \\ ${ }^{1}$ Department of Urology, Azienda Ospedaliero-Universitaria Policlinico S.Orsola-Malpighi, Bologna, Italy; \\ ${ }^{2}$ Department of Pathology, Azienda Ospedaliero-Universitaria Policlinico S.Orsola-Malpighi, Bologna, Italy; \\ ${ }^{3}$ Andrology Unit, Azienda Ospedaliero-Universitaria Policlinico S.Orsola-Malpighi, Bologna, Italy.
}

\begin{abstract}
Summary Spermatocytic Seminoma (SS) is less common than the Classic variant, as its incidence ranges between $1.3 \%$ and $2.3 \%$ of all seminomas. Generally SS is diagnosed in men older than 50 years. The Anaplastic variant of Spermatocytic Seminoma is characterized by an earlier onset when compared to SS, but a benign behavior in spite of its histological patterns similar to Classic Seminoma. We reported the first case of bilateral, largest and synchronous Anaplastic

Spermatocytic Seminoma, in a patient treated with radical orchifunicolectomy alone and with long-term follow-up.

The currently available data show that Anaplastic SS reveals a clinically benign behavior, and no distant metastases have been reported so far. A close surveillance after surgery could be considered a valid option in the management of this rare testicular neoplasm.
\end{abstract}

KEY WORDS: Testicular cancer; Spermatocytic seminoma; Anaplastic variant; Bilateral disease; Radical orchifunicolectomy

Submitted 5 November 2013; Accepted 31 December 2013

\section{AIM OF THE PAPER}

In this paper we report the seventh case of Anaplastic Spermatocytic Seminoma (SS) (1-14), the first with bilateral and synchronous presentation and unusual clinical features.

\footnotetext{
Discussion

Spermatocytic Seminoma (SS) is a rare germ cell tumor, characterized by a benign behavior with slow growth, and is generally localized in the testis; this tumor has a good long term prognosis, and is always controlled by one of the simplest intervention in urology, such as radical orchiectomy with nearly absent risks of complications contrarily to many other urological interventions $(15,16)$; in literature over 200 cases of SS have been described with only three cases of clearly established metastasis for the
}

conventional type of this tumor (8). It is universally accepted that the sarcomatous differentiation of SS is associated with an aggressive behavior, the presence of metastasis and a poor prognosis $(12,13,17,18)$, despite aggressive surgical and adjuvant treatments (19). On the other hand the anaplastic variant of SS is still poorly known, as in literature only six cases of monolateral tumor have been described so far $(6,9,14)$; this is the first case of bilateral, synchronous anaplastic SS described till today. Still remain ambiguities about its biological behavior and about the most appropriate diagnostic and therapeutic protocol. However, the present tumor is the largest one described $(20 \mathrm{~cm})$ with the longest period of observation (60 months before the surgical intervention plus 36 months of follow-up), which confirm the low risk of metastatic pattern. The main clinical and pathological features of the seven cases (six available in literature and our case) are reported in Supplementary Materials (Table 1). The mean age at the moment of the diagnosis was 45.8 years. This finding confirms the data reported by other authors regarding the early onset of anaplastic variant compared to the classical spermatocytic seminoma (6). Furthermore our patient underwent medical examination 5 years after the initial clinical presentation of the disease, which strengthens the hypothesis of an earlier manifestation of the disease. The testicular mass was always asymptomatic and characterized by a slow growth and absence of metastasis; in particular in our case the delay of the diagnosis allowed the tumor to grow disproportionately, reaching an enormous size ( $20 \mathrm{~cm}$ diameter in the left mass) occupying the entire testis, but the growth was limited to the parenchyma without invasion of the tunica albuginea with no lymph node or distant metastases. In all cases there was no intratubular germ cell neoplasia unclassified (IGCNU) associated. Serum markers $\alpha$-FP, $\beta$-HGB were negative in all cases. The markers commonly used for the diagnosis of Classic Seminoma (CS) were always negative but only in two cases has been observed the positivity for c-kit (CD117), which has also been reported to be positive in SS in some cases $(20,21)$; our case was the first with a focal positivity for PLAP and this is the first identification 
of this marker in an anaplastic variant of SS, even if isolated positivity for PLAP in classic SS has been reported (2022). All the patients underwent radical orchiectomy (RO), while the management after surgery was different but in any case there was the onset of metastasis or recurrence: In 3 cases RO was followed by radiotherapy applied to pelvic and retroperitoneal lymph nodes, in 2 cases the treatment was consolidated with 2 cycles of chemotherapy (carboplatin or cisplatin, etoposide and bleomycin), in two cases $\mathrm{RO}$ was followed by surveillance with clinical examination and imaging. Histological findings in anaplastic SS, such as areas with extensive necrosis, solid growth pattern, multiple mitotic figures, vascular and tunical invasion, and anaplastic features in lymph node metastasis in primary SS have been described by Albores-Saavedra et al. (6). According to these findings, the anaplastic variant of SS would seem to have a more aggressive behavior compared to the classical form, even if all the cases described in literature showed a benign behavior and a good prognosis comparable to that of typical SS. In the absence of a specific radiotracer such as for other urological malignancies (23) the PET18F-FDG plus contrast enhanced whole body CT seems the most appropriate follow-up behaviour.

\section{References}

1. Masson P. Etude sur le seminome. Rev Can Biol. 1946; 5:361-87.

2. Mostofi F, Sesterhenn I. Tumours of the testis and paratesticular tissue. In: World Health Organization Classification of Tumours: Pathology and Genetics-Tumours of the Urinary System and Male Genital Organs. Edited by JN Eble, G Sauter, JI Epstein and IA Sesterhenn. Lyon: International Agency for Research on Cancer (IARC) Press 2004; chapt 4, pp 217-278.

3. Looijenga LH, Stoop H, Hersmus R, et al. Genomic and expression profiling of human spermatocytic seminomas: pathogenetic implications international. Int J Androl. 2007; 30:328-35; discussion 335-6. Epub 2007 Jun 15.

4. Bomeisl PE, MacLennan GT. Spermatocytic Seminoma. Int J Androl. 2007; 177:734.

5. Stephenson AJ, Gilligan TD. Neoplasms of the testis. In: Walsh PC, Retik AB, Vaughan Jr ED, Wein AJ, editors. Campbell's Urology. 10th ed. Philadelphia (PA): Saunders. 2012; p. 840.

6. Albores-Saavedra J, Huffman H, Alvarado-Cabrero I, et al. Anaplastic variant of spermatocytic seminoma. Hum Pathol. 1996; 27:650-655.

7. Brunocilla E, Pultrone CV, Schiavina R, et al. Testicular sclerosing Sertoli cell tumor: an additional case and review of the literature. Anticancer Res. 2012; 32:5127-30.

8. Malizia M, Brunocilla E, Bertaccini A, et al. Liposarcoma of the spermatic-cord: description of two clinical cases and review of the literature. Arch Ital Urol Androl. 2005; 77:115-7.

9. Dundr P, Pesl M, et al. Anaplastic variant of spermatocytic seminoma. Pathol Res Pract. 2007; 203:621-4.

10. Chung PW, Bayley AJ, Sweet J, et al. Spermatocytic seminoma: a review. Eur Urol. 2004; 45:495.

11. Burke AP, Mostofi FK. Spermatocytic seminoma: a clinicopathologic study of 79 cases. J Urol Pathol. 1993; 1:21-32.

12. Narang V, Gupta K, Gupta A, et al. Rhabdomyosarcomatous differentiation in a spermatocytic seminoma with review of literature. Indian J Urol. 2012; 28:430-433.
13. Menon S, Karpate A, Desai D. Spermatocytic seminoma with rhabdomyosarcomatous differentiation: a case report with a review of the literature. J Cancer Res Ther. 2009; 5:213-5.

14. Lombardi $M$, Valli $M$, Brisigotti $M$, et alhttp://www.ncbi.nlm. nih.gov/pubmed?term=RosaiJ\%5BAuthor\%5DEcauthor=true Ecauthor_uid=21087978. Spermatocytic seminoma: review of the literature and description of a new case of the anaplastic variant. nt J Surg Pathol. 2011; 19:5-10.

15. Schiavina R, Borghesi M, Guidi M, et al. Perioperative complications and mortality after radical cystectomy when using a standardized reporting methodology. Clin Genitourin Cancer. 2013; 11:189-97.

16. Brunocilla E, Pultrone C, Pernetti R, et al. Preservation of the smooth muscular internal (vesical) sphincter and of the proximal urethra during retropubic radical prostatectomy: description of the technique. Int J Urol. 2012; 19:783-5.

17. Chelly I, Mekni A, Gargouri MM, et al. Spermatocytic seminoma with rhabdomyosarcomatous contingent. Prog Urol. 2006; 16:218-20.

18. True LD, Otis CN, Delprado W, et al. Spermatocytic seminoma of testis with sarcomatous transformation. A report of five cases. Am J Surg Pathol. 1988; 12:75-82.

19. Robinson A, Bainbridge T, Kollmannsberger C. A spermatocytic seminoma with rhabdomyosarcoma transformation and extensive metastases. Am J Clin Oncol. 2007; 30:440-1.

20. Cummings OW, Ulbright TM, Eble JN, et al. Spermatocytic semonima: an immunohistochemical study, Hum Pathol. 1994; 25:54-59.

21. Kraggerud SM, Berner A, Bryne M, et al. Spermatocytic seminoma as compared to classical seminoma: an immunohistochemical and DNA flow cytometric study, APMIS 1999; 107:297-302.

22. Dekker I, Rozeboom T, Delemarre J, et al. Placental-like alkaline phosphatase and DNA flow cytometry in spermatocytic seminoma, Cancer 1992; 69:993-996.

23. Nanni C, Schiavina R, Boschi S, et al. Comparison of 18FFACBC and 11C-choline PET/CT in patients with radically treated prostate cancer and biochemical relapse: preliminary results. Eur J Nucl Med Mol Imaging. 2013; 40(Suppl 1):S11-7.

Case report, Table and Figures are posted in Supplementary materials on www.aiua.it

\section{Correspondence}

Giorgio Gentile, MD - dr.giorgio.gentile@gmail.com

Riccardo Schiavina, MD, Assistant Professor - rschiavina@yahoo.it

Marco Borghesi, MD - mark.borghesi@gmail.com (Corresponding Author)

Ziv Zukerman, MD - ziv.zukerman@gmail.com

Matteo Cevenini, MD - matteoceve@gmail.com

Valerio Vagnoni, MD - vagno07@libero.it

Daniele Romagnoli, MD - danieleromagnoli@hotmail.it

Giuseppe Martorana, MD, Professor - giuseppe.martorana@unibo.it

Eugenio Brunocilla, MD, Associate Professor - eugenio.brunocilla@unibo.it Department of Urology, Azienda Ospedaliero-Universitaria Policlinico S.Orsola-Malpighi, Palagi 9 street, 40138 Bologna, Italy

Francesca Giunchi, MD - francesca.giunchi@aosp.bo.it

Department of Pathology, Azienda Ospedaliero-Universitaria

Policlinico S.Orsola-Malpighi, Palagi 9 street, 40138 Bologna, Italy

Alessandro Franceschelli, MD, PhD - alessandro.franceschelli@aosp.bo.it Fulvio Colombo, MD - fulvio.colombo@aosp.bo.it

Andrology Unit, Azienda Ospedaliero-Universitaria

Policlinico S.Orsola-Malpighi, Palagi 9 street, 40138 Bologna, Italy 